\title{
COMMUTATIVE GROUP ALGEBRAS
}

\author{
BY \\ WARREN MAY
}

Let $R$ be a commutative ring with identity. If $G$ is a group, then one can form the group algebra of $G$ over $R$ which we shall denote by $R[G]$. One can then ask the basic question, how much information about the group $G$ can be deduced from the $R$-algebra $R[G]$ ? A slightly different formulation is to assume that $G$ and $H$ are groups with $R[G]$ isomorphic to $R[H]$ as $R$-algebras and to ask what relations exist between $G$ and $H$. A discussion of the problem with references can be found in Curtis-Reiner [2, p. 262]. There they prove the early result of Higman [4] that if $R$ is the ring of integers and the groups are finite abelian, then isomorphism of the group algebras implies isomorphism of the groups. Later work of Perlis-Walker [8] and Deskins [3] takes up the problem over fields. One result is that if we take $R$ to be the rational numbers, then the group algebras determine finite abelian groups.

There are difficulties in trying to apply the methods of group representations to other situations, particularly if the characteristic of $R$ is finite. However, if the group algebra is commutative, i.e., the group is abelian, then in case $R$ has characteristic $p$, one has the Frobenius endomorphism of $R[G]$. In this paper we show that systematic application of this mapping enables one to deduce fairly broad conclusions about abelian groups with isomorphic group algebras over commutative rings. In fact the group modulo its torsion subgroup is completely determined. To deduce conclusions about a $p$-primary component of its torsion subgroup however, we must require that $R$ behaves sufficiently well with respect to the prime $p$. More precisely, one requires that $p$ is not invertible in $R$. Under this condition, the maximal divisible subgroup and the Ulm invariants of the $p$-primary component are determined. A complete statement of results is contained in the theorem in the last section. In the middle two sections we work over fields of various types and then generalize to arbitrary commutative rings later.

1. Preliminaries. It is worthwhile to prove several standard propositions on group algebras since they will be used repeatedly in the succeeding sections. Let $R$ be a fixed commutative ring with identity. Then the formation of group algebras over $R$ gives a functor from the category of groups to the category of $R$-algebras. If $f: G \rightarrow H$ is a homomorphism of groups, then $f$ induces an $R$-algebra homomorphism $f^{\prime}: R[G] \rightarrow R[H]$ by $f^{\prime}\left(\sum r_{g} g\right)=\sum r_{g} f(g)$. If we identify $R$ with the group algebra of a fixed trivial group, then the homomorphism of $G$ onto this trivial

Received by the editors February 16, 1967 and, in revised form, November 27, 1967. 
group induces an $R$-algebra homomorphism of $R[G]$ onto $R$ called the augmentation map and denoted $i: R[G] \rightarrow R$. Then $i$ is given by $i\left(\sum r_{g} g\right)=\sum r_{g}$. The kernel of $i$ is called the augmentation ideal of $R[G]$. Our first proposition says that if we have an arbitrary $R$-algebra map of $R[G]$ into $R$ then one can change the group algebra by an automorphism so that the given map becomes the augmentation map. A reference in the literature for this result is Losey [6, Theorem 2.3].

Proposition 1. Let $G$ be a group and $i$ the augmentation map on $R[G]$. Suppose $T$ is an $R$-algebra such that $T \cong R[G]$ as $R$-algebras. Assume we are given an $R$ algebra map $i^{\prime}: T \rightarrow R$. Then there exists an $R$-algebra isomorphism $\varphi: T \rightarrow R[G]$ such that $i \circ \varphi=i^{\prime}$.

Proof. Equivalently, given $i^{\prime}: R[G] \rightarrow R$, we may ask for an automorphism $\varphi$ of $R[G]$ such that $i \circ \varphi=i^{\prime}$. For $g \in G$, put $r_{g}=i^{\prime}(g)$. Define $\varphi: G \rightarrow R[G]$ by $\varphi(g)=r_{g} g$. Then $\varphi$ is a homomorphism and so by the mapping property of group algebras, $\varphi$ extends to an $R$-algebra map $\varphi: R[G] \rightarrow R[G]$. It is easy to show $\varphi$ has an inverse (since $\left(r_{g}\right)^{-1}=r_{g}-1$ ) and that $i \circ \varphi=i^{\prime}$.

COROLLARY 1. Let $G_{1}$ and $G_{2}$ be groups and $M_{1}$ and $M_{2}$ the augmentation ideals of $R\left[G_{1}\right]$ and $R\left[G_{2}\right]$ respectively. If $R\left[G_{1}\right] \cong R\left[G_{2}\right]$ as $R$-algebras, then we may choose an isomorphism $\varphi$ such that $\varphi\left(M_{1}\right)=M_{2}$.

At this point the author would like to thank the referee for his helpful remarks and for suggesting the next two corollaries. Suppose that $\varphi: R\left[G_{1}\right] \rightarrow R\left[G_{2}\right]$ is an isomorphism commuting with the augmentation maps, and that $A$ is an $R$-module. Let $G_{1}$ act on $A$ trivially and let $G_{2}$ act on $A$ by the action induced by $\varphi$. It then follows that $G_{2}$ acts trivially on $A$.

Corollary 2. Let $G_{1}$ and $G_{2}$ be groups and $A$ an $R$-module on which we let $G_{1}$ and $G_{2}$ act trivially. Assume $R\left[G_{1}\right] \cong R\left[G_{2}\right]$ as $R$-algebras. Then $H^{n}\left(G_{1}, A\right) \cong$ $H^{n}\left(G_{2}, A\right)$ and $H_{n}\left(G_{1}, A\right) \cong H_{n}\left(G_{2}, A\right)$ for all $n \geqq 0$ (where homology and cohomology are taken over $R\left[G_{1}\right]$ or $\left.R\left[G_{2}\right]\right)$.

Applying this corollary to the integers and recalling that $H_{1}(G, Z)$ is the abelianization of $G$, we obtain

COROLlaRY 3. Two groups having isomorphic group algebras over the integers have isomorphic abelianizations. Hence, if the two groups are abelian, then they are isomorphic.

The next proposition is stated in a slightly more general form than one would normally use, but this will simplify later applications. For comparisons, see Connell [1, Proposition 1] and Gruenberg [5, Lemma 2]. We shall use the letter $e$ to denote identity elements of groups. Presumably no confusion will arise from using it to denote the identity elements in different groups simultaneously. 
Proposition 2. Let $G$ be a group, $S$ a subset of $G$, and $H$ the normal subgroup of $G$ generated by $S$. Let I be the two-sided ideal of $R[G]$ generated by the set of elements $\{s-e \mid s \in S\}$. Let $j: R \rightarrow R$ be an injective ring homomorphism and suppose $f: G \rightarrow G / H$ is the natural map. Then $j$ and $f$ induce a ring homomorphism $\varphi: R[G]$ $\rightarrow R[G / H]$ by $\varphi\left(\sum r_{g} g\right)=\sum j\left(r_{g}\right) f(g)$. The kernel of $\varphi$ is $I$.

Proof. For $s \in S$ it is clear that $\varphi(s-e)=0$, hence $I \subseteq(\operatorname{kernel} \varphi)$. Suppose that $g_{1}-e$ and $g_{2}-e$ are both in $I$. Then $g_{1} g_{2}-e=\left(g_{1}-e\right)\left(g_{2}-e\right)+\left(g_{1}-e\right)+\left(g_{2}-e\right)$ is in $I$. Also if $(g-e) \in I$, then $g^{-1}-e=-g^{-1}(g-e)$ is in $I$. Finally if $(g-e) \in I$ and $b \in G$, then $b g b^{-1}-e=b(g-e) b^{-1}$ is in $I$. It follows now that $\{h-e \mid h \in H\} \subseteq I$.

Choose $\left\{g_{i}\right\}$ to be a complete family of coset representatives of $H$ in $G$. Let $\alpha \in R[G]$, say $\alpha=\sum_{i, h} r_{i, h} g_{i} h$ where $h \in H$. Then

$$
\varphi(\alpha)=\sum_{i, h} j\left(r_{i, h}\right) f\left(g_{i}\right)=\sum_{i} f\left(g_{i}\right)\left(\sum_{h} j\left(r_{i, h}\right)\right)=\sum_{i} f\left(g_{i}\right) j\left(\sum_{h} r_{i, h}\right) .
$$

Hence $\varphi(\alpha)=0$ implies that $j\left(\sum_{h} r_{i, h}\right)=0$ for all $i$ and so $\sum_{h} r_{i, h}=0$ for all $i$. It follows that $\sum_{h \neq e}\left(-r_{i, h}\right)=r_{i, e}$ and so $\sum_{h} r_{i, h} h=\sum_{h \neq e} r_{i, h}(h-e)$ which is contained in $I$. Therefore $\alpha=\sum_{i} g_{i}\left(\sum_{h} r_{i, h} h\right)$ is contained in $I$. Hence (kernel $\left.\varphi\right) \subseteq I$.

COROLlary 4. The augmentation ideal of $R[G]$ is generated by $\{g-e \mid g \in G\}$.

CoROllary 5. Let $H$ be a subgroup of $G$ and $M$ the augmentation ideal of $R[G]$. Then, regarding $R[H]$ as a subalgebra of $R[G]$, the augmentation ideal of $R[H]$ is $M \cap R[H]$. If H is normal and I is the two-sided ideal of $R[G]$ generated by $M \cap R[H]$, then $R[G] / I \cong R[G / H]$.

Proof. It is clear that $M \cap R[H]$ is the augmentation ideal of $R[H]$. Since $\{h-e \mid h \in H\}$ generates $M \cap R[H]$ as an ideal in $R[H]$, the same set generates $I$ as an ideal in $R[G]$. The claim now follows.

2. The torsion-free case. By a localized polynomial ring we shall mean a polynomial ring over a commutative ring with identity which is localized at the multiplicative monoid of monomials. The first two lemmas we prove can be traced back to Higman [4, Theorem 12 and 13].

Lemma 1. Let $F$ be a field and $G$ an abelian group. Then $F[G]$ has nontrivial zero divisors if and only if $G$ is not torsion-free.

Proof. Any two elements of $F[G]$ are contained in a subalgebra of form $F\left[G_{1}\right]$ where $G_{1}$ is some finitely generated subgroup of $G$. Then $G$ torsion-free implies $G_{1}$ is a free abelian group, hence $F\left[G_{1}\right]$ is isomorphic to a localized polynomial ring over $F$ in a finite number of variables. Therefore $F\left[G_{1}\right]$ is a domain, hence $F[G]$ must be a domain.

If $G$ is not torsion-free, then $G$ has a nontrivial finite subgroup, call it $K$. Put $\alpha=\sum_{x \in K} x$. Then if $|K|=n$, we see $\alpha^{2}=n \alpha$, and so $\alpha(\alpha-n)=0$. Hence both $\alpha$ and $\alpha-n$ nonzero implies $F[G]$ has nontrivial zero divisors. 
LEMMA 2. Let $F$ be a field and $G$ and $H$ torsion-free abelian groups such that $F[G] \cong F[H]$ as $F$-algebras. Then $G \cong H$.

Proof. Let $F^{*}$ denote the multiplicative group of $F$. We claim that the group of units of $F[G]$ is $U=\left\{r g \mid r \in F^{*}, g \in G\right\}$. It is clear $U$ is a subgroup of the group of units. If $\alpha \in F[G]$, then $\alpha \in F\left[G_{1}\right]$ for some finitely generated subgroup $G_{1}$ of $G$. Now regarding $F\left[G_{1}\right]$ as a subalgebra of $F[G]$, it suffices to show that the units of $F\left[G_{1}\right]$ have the proper form. As in the preceding proof, it follows that $F\left[G_{1}\right]$ is isomorphic to a localized polynomial ring over $F$ in $n$ variables by an $F$-isomorphism which identifies $G_{1}$ with the group of monomials. But it is an easy lemma that for localized polynomial rings over fields, the group of units is generated by the nonzero scalars and the monomials. Hence the group of units in $F\left[G_{1}\right]$ is $\left\{r g \mid r \in F^{*}, g \in G_{1}\right\}$. Therefore $U$ is precisely the group of units of $F[G]$. Now $U$ is the inner direct product of $F^{*}$ and $G$. Similarly, if $V$ is the group of units in $F[H], V$ is the inner direct product of $F^{*}$ and $H$. If $\varphi: F[G] \rightarrow F[H]$ is an $F$ algebra isomorphism, then $\varphi(U)=V$ and $\varphi\left(F^{*}\right)=F^{*}$. Hence $G \cong H$.

Let $p$ be a prime and let $S$ be either an abelian group written multiplicatively or a commutative ring with identity of characteristic $p$. We shall define the map $\theta: S \rightarrow S$ by $\theta(s)=s^{p}$ for $s \in S$ and call it the Frobenius endomorphism of $S$. It is either a group homomorphism or a ring homomorphism as the case may be. Again, no confusion should result, since each context will make clear what $p$ and the domain of $\theta$ are.

LemMa 3. Let $F$ be a field of characteristic $p$ and $G$ and $H$ abelian groups with p-primary components $G(p)$ and $H(p)$ respectively. Assume $F[G] \cong F[H]$ as $F$ algebras. Then $F[G / G(p)] \cong F[H / H(p)]$ as $F$-algebras.

Proof. For a positive integer $n$, let $\theta^{n}$ be the $n$-fold iteration of $\theta$. Consider $\theta^{n}: F[G] \rightarrow F[G]$. Then if $\alpha \in F[G]$, say $\alpha=\sum r_{g} g$, we have $\theta^{n}(\alpha)=\sum \theta^{n}\left(r_{g}\right) \theta^{n}(g)$. Hence the action of $\theta^{n}$ on $F[G]$ is induced by the actions on $F$ and $G$ individually. Since $\theta^{n}$ is injective on $F$, we may apply Proposition 2. If $I_{n}$ is the kernel of $\theta^{n}$ acting on $F[G]$ and $G_{n}$ is the kernel of $\theta^{n}$ acting on $G$, we have that $I_{n}$ is generated by $\left\{g-e \mid g \in G_{n}\right\}$. Clearly $\left\{I_{n}\right\}$ is an increasing sequence of ideals. Put $I=\bigcup_{n=1}^{\infty} I_{n}$.

Now suppose that we have defined ideals $\left\{J_{n}\right\}$ and $J$ in $F[H]$ in the same way. Let $\varphi: F[G] \rightarrow F[H]$ be an $F$-algebra isomorphism. Then it is clear that $\varphi\left(I_{n}\right)=J_{n}$ for all $n$ and hence $\varphi(I)=J$. Therefore $F[G] / I \cong F[H] / J$ as $F$-algebras. But $I$ is generated by $\left\{g-e \mid g \in \bigcup_{n} G_{n}\right\}=\{g-e \mid g \in G(p)\}$. Therefore $F[G] / I \cong F[G / G(p)]$. Similarly for $F[H]$.

We say an algebra over a field is algebraic if every element of the algebra is contained in a finite dimensional subalgebra. This is equivalent to saying that every element satisfies a nontrivial polynomial with coefficients in the field.

Proposition 3. Let $G$ and $H$ be abelian groups with torsion subgroups $G_{0}$ and $H_{0}$ respectively. Let $F$ be an algebraically closed field. Assume that $F[G] \cong F[H]$ as $F$ algebras. Then $F\left[G / G_{0}\right] \cong F\left[H / H_{0}\right]$ as $F$-algebras. 
Proof. If $F$ has characteristic $p$, then $F[G / G(p)] \cong F[H / H(p)]$, hence we may assume in this case that $G$ and $H$ have trivial $p$-primary components.

We claim that $F\left[G_{0}\right]$ is the maximal algebraic subalgebra of $F[G]$, that is, it consists of all the elements of $F[G]$ algebraic over $F$. Every element of $F\left[G_{0}\right]$ is contained in a subalgebra of form $F[A]$ where $A$ is a finitely generated subgroup of $G_{0}$ and hence finite. Therefore $F\left[G_{0}\right]$ is certainly an algebraic subalgebra.

Now suppose that $\alpha \in F[G]$ is contained in a finite dimensional subalgebra. Then that subalgebra is contained in a subalgebra of form $F\left[G_{1}\right]$ where $G_{1}$ is a finitely generated subgroup of $G$. Let $A$ be the torsion subgroup of $G_{1}$. Then we may write $G_{1}$ as the inner direct product of $A$ and $T$ where $T$ is a free subgroup of $G_{1}$ of rank $n$ for some $n$. Since we may assume the characteristic of $F$ does not divide the order of $A$, we have that $F[A]$ is semisimple, and since $A$ is abelian and $F$ is algebraically closed, we must have $F[A] \cong \bigoplus_{i=1}^{m} F$ as $F$-algebras. Therefore, since the group algebra functor takes direct sums of abelian groups into tensor products of algebras, we have

$$
F\left[G_{1}\right]=F[A T] \cong F[A] \otimes_{F} F[T] \cong \bigoplus_{i=1}^{m} F[T] .
$$

With respect to this direct sum decomposition, suppose $\alpha$ has form $\alpha=\left(f_{1}, \ldots, f_{m}\right)$ where each $f_{i} \in F[T]$. Now $F[T]$ is isomorphic to a localized polynomial ring over $F$ in $n$ variables. Take an $F$-basis for the finite dimensional subalgebra in which $\alpha$ is contained. Then looking at the projection of this basis onto each summand, $F[T]$, we see only finitely many monomials in $F[T]$ are involved nontrivially in expressing the projections. But for $k \geqq 1, \alpha^{k}=\left(f_{1}^{k}, \ldots, f_{m}^{k}\right)$ is contained in this subalgebra, hence each $f_{i}^{k}$ must only involve a finite number of monomials nontrivially for all $k$. It follows that $f_{i}$ must be constant for all $i$. Now the constants in $F[T]$ are characterized as the subalgebra generated by the identity element. Hence to say that every component of $\alpha$ is a constant is to say that $\alpha$ is contained in the subalgebra of $F\left[G_{1}\right]$ generated by the idempotents. But this subalgebra is $F[A]$ since $F[A]$ contains $m$ linearly independent idempotents. Therefore $\alpha \in F[A] \subseteq F\left[G_{0}\right]$ and so $F\left[G_{0}\right]$ is the maximal algebraic subalgebra of $F[G]$. A similar statement applies to $F\left[H_{0}\right]$.

Let $\varphi: F[G] \rightarrow F[H]$ be an $F$-isomorphism. Then if $M_{G}$ and $M_{H}$ are the augmentation ideals of $F[G]$ and $F[H]$ respectively, we may assume that $\varphi\left(M_{G}\right)=M_{H}$. But $\varphi\left(F\left[G_{0}\right]\right)=F\left[H_{0}\right]$ since the notion of algebraic algebra is preserved under $F$-maps. Define $I_{G}$ to be the ideal of $F[G]$ generated by $M_{G} \cap F\left[G_{0}\right]$. If $I_{H}$ is defined in the same way, then $\varphi\left(I_{G}\right)=I_{H}$. But $F[G] / I_{G} \cong F\left[G / G_{0}\right]$ by Corollary 5, and similarly for $H$. Hence $\varphi$ allows us to deduce $F\left[G / G_{0}\right] \cong F\left[H / H_{0}\right]$.

Application of Lemma 2 yields

COROLlary 6. Let $F$ be an algebraically closed field and $G$ and $H$ abelian groups with torsion subgroups $G_{0}$ and $H_{0}$. Assume that $F[G] \cong F[H]$ as F-algebras. Then $G / G_{0} \cong H / H_{0}$. 
3. The torsion case. For $p$ a prime, we shall let $\Gamma_{\infty}$ denote the $p$-primary component of the group of roots of unity and let $\Gamma$ denote the subgroup of $p$ th roots of unity. Let $A$ be an indexing set and consider $\sum_{A} \Gamma_{\infty}$. For each $a \in A$, let $g_{a}$ be a fixed generator of $\Gamma$ in the copy of $\Gamma_{\infty}$ corresponding to $a$.

LEMMA 4. Let $F$ be a field of characteristic $p$ and let $K$ be the kernel of $\theta$ acting on $F[G]$ where $G \cong \sum_{A} \Gamma_{\infty}$. Let $M$ be the augmentation ideal of $F[G]$ and assume that $|A|$ is finite. Then $K / M K$ is a vector space over $F$ of dimension $|A|$.

Proof. Let - denote reduction modulo $M$. Then the augmentation map induces an $F$-isomorphism $(F[G])^{-} \cong F$. Since $\theta$ is injective on $F$, by Proposition 2 we see that $K$ is generated by $\left\{g_{a}-e \mid a \in A\right\}$ since $\sum_{A} \Gamma$ is the kernel of $\theta$ acting on $\sum_{A} \Gamma_{\infty}$, and it is generated by $\left\{g_{a}\right\}$. Moreover, this is clearly a minimal set of generators. Now $F[G]$ is a local ring (this follows since $M$ is maximal and every element of $M$ is nilpotent) and $K$ a finitely generated $F[G]$-module. Hence by an application of Nakayama's lemma (see Nagata $[7,5.1]$ ) we conclude that $|A|$ is the dimension of $\bar{K}$ over $F$.

Proposition 4. Let $G_{d}$ and $G_{d}^{\prime}$ be divisible p-primary abelian groups and let $H$ and $H^{\prime}$ be abelian groups with trivial p-primary components. Let $F$ be an algebraically closed field of characteristic p. Assume $F\left[G_{d} \times H\right] \cong F\left[G_{d}^{\prime} \times H^{\prime}\right]$ as F-algebras. Then $G_{d} \cong G_{d}^{\prime}$.

Proof. (1) Let the given isomorphism be $\varphi$ and let $M$ and $M^{\prime}$ be the augmentation ideals of the two group algebras. Let $K$ and $K^{\prime}$ be the respective kernels of $\theta$ acting on the two algebras. Then we may assume $\varphi(M)=M^{\prime}$, and hence $K / M K \cong K^{\prime} / M^{\prime} K^{\prime}$ since $\varphi(K)=K^{\prime}$. Now $G_{d} \cong \sum_{A} \Gamma_{\infty}$ and $G_{d}^{\prime} \cong \sum_{A^{\prime}} \Gamma_{\infty}$ for some indexing sets $A$ and $A^{\prime}$. We shall show that $\operatorname{dim}_{F}(K / M K)=|A|$. Since a similar statement applies to $\left|A^{\prime}\right|$, we will be finished.

(2) As in the proof of the preceding lemma, we have that $K$ is generated by $\left\{g-e \mid g \in G_{d} \times H, \theta(g)=e\right\}$. But since $H$ has trivial $p$-primary component, we see this set is just $\left\{g-e \mid g \in \sum_{A} \Gamma\right\}$, where we have replaced $G_{d}$ by $\sum_{A} \Gamma_{\infty}$. Hence $\left\{g_{a}-e \mid a \in A\right\}$ will generate $K$. As before, if - denotes reduction modulo $M$, then $\left\{\left(g_{a}-e\right)^{-}\right\}$generates $\bar{K}$ as an $F$-module. Again the problem is reduced to showing this is an independent set of generators.

(3) Assume in this part that $|A|$ is finite and that $H$ is finitely generated. Under these assumptions we shall prove that $\operatorname{dim}_{F}(K / M K)=|A|$ and hence $\left\{\left(g_{a}-e\right)^{-}\right\}$ is an independent set under these conditions. The procedure will be to reduce the problem to one to which the lemma may be applied.

Now $H \cong H_{0} \times T$ where $H_{0}$ is a finite abelian group with trivial $p$-primary component and $T$ is a free abelian group of rank $n$ for some $n$. Therefore one has

$$
F\left[G_{d} \times H\right] \cong F\left[H_{0}\right] \otimes_{F} F\left[G_{d}\right] \otimes_{F} F[T] .
$$


Since $F$ is algebraically closed, as in a previous proof, we have $F\left[H_{0}\right] \cong \bigoplus_{i=1}^{m} F$. Put $L=F\left[G_{d}\right]$. Then $F\left[G_{d}\right] \otimes_{F} F[T]$ is isomorphic to a localized polynomial ring over $L$ in $n$ variables. So one has $F\left[G_{d} \times H\right] \cong \bigoplus_{i=1}^{m} Q$ where

$$
Q=L\left[t_{1}, \ldots, t_{n}, t_{1}^{-1}, \ldots, t_{n}^{-1}\right]
$$

Next we must retrieve $M$ and $K$. Since the kernel of $\theta$ is invariant under isomorphism, we see that $K \cong \bigoplus_{i=1}^{m} K_{1}$ where $K_{1}$ is the kernel of $\theta$ applied to $Q$. Let $f \in Q$, say $f=\sum_{(i)} b_{(i)} t_{1}^{i_{1}} \cdots t_{n}^{i_{n}}$ where $(i)$ varies over $n$-tuples of integers. Then $\theta(f)=f^{p}=\sum_{(i)} b_{(i)}^{p} t_{i}^{p i_{1}} \cdots t_{n}^{p i_{n}}$. Now $\theta$ gives an injection of the family of monomials in $Q$ into itself, hence $\theta(f)=0$ if and only if $b_{(i)}^{p}=0$ for all $(i)$. That is, if and only if $b_{(i)} \in K_{0}$ where $K_{0}$ is the kernel of $\theta$ in $L$. Therefore $K_{1}$ is the ideal in $Q$ generated by $K_{0}$. Hence we may write $K_{1}=\bigoplus_{(i)} K_{0} t_{1}^{i_{1}} \cdots t_{n}^{i_{n}}$. Now all we know of $M$ is that it is isomorphic to some maximal ideal in $\oplus_{i=1}^{m} Q$. Therefore we may assume this ideal has form $M_{1} \oplus\left(\oplus_{i=2}^{m} Q\right)$ where $M_{1}$ is some maximal ideal in the first copy of $Q$. Hence we have that $K / M K \cong K_{1} / M_{1} K_{1}$. We are reduced to looking at one copy of $Q$ and showing that $\operatorname{dim}_{F}\left(K_{1} / M_{1} K_{1}\right)=|A|$.

As in the proof of the lemma, $L$ is a local ring whose maximal ideal, call it $M_{0}$, consists of nilpotent elements. It follows that $M_{1} \cap L=M_{0}$ since $M_{1}$ must contain all nilpotent elements, but cannot contain a unit. We shall show that $K_{1} / M_{1} K_{1}$ $\cong K_{0} / M_{0} K_{0}$ and so reduce the problem entirely to $L=F\left[G_{d}\right]$. But Lemma 4 will then apply to give $\operatorname{dim}_{F}\left(K_{0} / M_{0} K_{0}\right)=|A|$. Hence because of the isomorphisms we will have $\operatorname{dim}_{F}(K / M K)=|A|$.

Let $I$ be the ideal of $Q$ generated by $M_{0}$. Then $Q / I \cong F\left[t_{1}, \ldots, t_{n}, t_{1}^{-1}, \ldots, t_{n}^{-1}\right]$ and since $I \subseteq M_{1}$, it follows that $M_{1}$ is the inverse image of a maximal ideal in $Q / I$ under the natural map. But since $F$ is algebraically closed and $Q / I$ is a localized polynomial ring over $F$, it follows that such a maximal ideal in $Q / I$ is generated by $t_{1}-b_{1}^{\prime}, \ldots, t_{n}-b_{n}^{\prime}$ where all $b_{1}^{\prime} \in F$ are nonzero (since our polynomial ring is localized at $\left.t_{1}, \ldots, t_{n}\right)$. Let $b_{1}, \ldots, b_{n}$ be preimages of $b_{1}^{\prime}, \ldots, b_{n}^{\prime}$ in $L$. Then $b_{1}, \ldots$, $b_{n}$ are all units in $L$ and $M_{1}$ is generated by $M_{0}, t_{1}-b_{1}, \ldots, t_{n}-b_{n}$ as an ideal in $Q$.

We shall establish that $K_{0}+M_{1} K_{1}=K_{1}$ and that $M_{0} K_{0}=M_{1} K_{1} \cap K_{0}$. From this it will follow that

$$
K_{1} / M_{1} K_{1} \cong K_{0} /\left(M_{1} K_{1} \cap K_{0}\right) \cong K_{0} / M_{0} K_{0}
$$

As remarked earlier, this will suffice to finish the proof. First consider $M_{0} K_{0}$ $=M_{1} K_{1} \cap K_{0}$. Clearly $M_{0} K_{0} \subseteq M_{1} K_{1} \cap K_{0}$. Now $M_{1} K_{1} \cap K_{0}$ may be interpreted as all constant polynomials in $M_{1} K_{1}$. Let $f \in M_{1} K_{1}$ be a constant polynomial. Let $\Delta$ be the substitution homomorphism on $Q$ given by $\Delta\left(t_{i}\right)=b_{i}$ for $i=1, \ldots, n$. Then from the structure of $M_{1}$ and $K_{1}$ we see that $\Delta\left(M_{1}\right)=M_{0}$ and $\Delta\left(K_{1}\right)=K_{0}$, hence $\Delta(f) \in M_{0} K_{0}$. But $f$ constant implies $\Delta(f)=f$. Hence $f \in M_{0} K_{0}$ and so $M_{1} K_{1} \cap K_{0} \subseteq M_{0} K_{0}$. 
Now consider $K_{0}+M_{1} K_{1}=K_{1}$. Clearly $K_{0}+M_{1} K_{1} \subseteq K_{1}$. Let $f \in K_{1}$ have form $f=\sum_{(i)} c_{(i)} t_{1}^{i_{1}} \cdots t_{n^{i}}$ with $c_{(i)} \in K_{0}$ for all $(i)$. Fix an $n$-tuple $(i)$ and suppose that, say, $i_{1}>0$. Since $t_{1}-b_{1} \in M_{1}$ and $c_{(i)} \in K_{1}$, it follows that

$$
c_{(i)}\left(t_{1}-b_{1}\right) t_{1}^{i_{1}-1} t_{2}^{i_{2}} \cdots t_{n}^{i_{n}} \in M_{1} K_{1} .
$$

But this element is just $c_{(i)} t_{1}^{i_{1}} \cdots t_{n^{n}}^{i_{n}}-c_{(i)} b_{1} t_{1}^{i_{1}-1} t_{2}^{i_{2}} \cdots t_{n^{n}}^{i_{n}}$. Hence we may "correct" $f$ by an element of $M_{1} K_{1}$ and replace the monomial $c_{(i)} t_{1}^{i_{1}} \cdots t_{n}^{i_{n}}$ by one of lower degree. If $i_{1}<0$, we can do the same by using $\left(t_{1}^{-1}-b_{1}^{-1}\right) \in M_{1}$ (note $t_{1}^{-1} \dot{\epsilon} Q$ and $b_{1}$ a unit make this possible). By an induction, one can add an element of $M_{1} K_{1}$ to $f$ to get a constant polynomial, i.e. a member of $K_{0}$. Hence $K_{1} \subseteq M_{1} K_{1}+K_{0}$.

(4) Now let $|A|$ and $H$ be arbitrary. We must show the set $\left\{\left(g_{a}-e\right)^{-}\right\}$is $F$ independent. If not, then a nontrivial $F$-linear combination of $\left\{g_{a}-e\right\}$, call it $\alpha$, lies in $M K$. Let $\alpha=\sum \beta_{i} \gamma_{i}$ where $\beta_{i} \in M, \gamma_{i} \in K$ for all $i$. Then one can choose a finite subset $A^{\#} \subseteq A$ and a finitely generated subgroup $H^{\#} \subseteq H$ such that $\beta_{i}$ and $\gamma_{i}$ belong to the subalgebra $F\left[\left(\sum_{A^{\#}} \Gamma_{\infty}\right) \times H^{\#}\right]$ for all $i$, and if $g_{a}-e$ is involved nontrivially in $\alpha$, then $a \in A^{\#}$. But it follows that each $\beta_{i}$ will lie in the augmentation ideal, $M^{\#}$, of this subalgebra, and that each $\gamma_{t}$ will lie in the kernel of $\theta$, call it $K^{\#}$, acting on the subalgebra. Hence $\alpha \in M^{\#} K^{\#}$. Therefore we have a nontrivial linear combination of $\left\{g_{a}-e \mid a \in A^{\#}\right\}$ which lies in $M^{\#} K^{\#}$. This contradicts the result of part (3) applied to the subalgebra.

Let $F$ be a field of characteristic $p$ and $T$ a commutative $F$-algebra. For each ordinal number $\alpha$, define $T_{\alpha}$ inductively as follows. Let $T_{1}=T$ and if $T_{\alpha}$ is defined, put $T_{\alpha+1}=\theta\left(T_{\alpha}\right)$. If $\alpha$ is a limit ordinal and $T_{\beta}$ is defined for all $\beta<\alpha$, put $T_{\alpha}=$ $\bigcap_{\beta<\alpha} T_{\beta}$. Then $\left\{T_{\alpha}\right\}$ is a decreasing family of subrings of $T$. If $F$ is perfect, then each $T_{\alpha}$ is also an $F$-subalgebra. Put $T_{\infty}=\bigcap_{\alpha} T_{\alpha}$. If $G$ is an abelian group, then replacing $T$ by $G$, we can define $\left\{G_{\alpha}\right\}$ and $G_{\infty}$ similarly.

COROLlary 7. Let $F$ be an algebraically closed field of characteristic p. Let $G$ and $G^{\prime}$ be abelian groups and let $G(p)_{d}$ and $G^{\prime}(p)_{d}$ denote the maximal divisible subgroups of their p-primary components respectively. Assume that $F[G] \cong F\left[G^{\prime}\right]$ as F-algebras. Then $G(p)_{d} \cong G^{\prime}(p)_{d}$.

Proof. Put $T=F[G]$ and $T^{\prime}=F\left[G^{\prime}\right]$. Then $T \cong T^{\prime}$ implies $T_{\alpha} \cong T_{\alpha}^{\prime}$ as $F$-algebras for all $\alpha$, and also $T_{\infty} \cong T_{\infty}^{\prime}$. We claim that $T_{\alpha}=F\left[G_{\alpha}\right]$ for all $\alpha$. It is true for $\alpha=1$. If $T_{\alpha}=F\left[G_{\alpha}\right]$ for some ordinal $\alpha$, then $T_{\alpha+1}=\theta\left(T_{\alpha}\right)=\theta\left(F\left[G_{\alpha}\right]\right)=F\left[\theta\left(G_{\alpha}\right)\right]=F\left[G_{\alpha+1}\right]$ since $F$ is perfect. If $\alpha$ is a limiting ordinal and $\beta<\alpha$ implies $T_{\beta}=F\left[G_{\beta}\right]$, then $T_{\alpha}=\bigcap_{\beta<\alpha} T_{\beta}=\bigcap_{\beta<\alpha} F\left[G_{\beta}\right]=F\left[\bigcap_{\beta<\alpha} G_{\beta}\right]=F\left[G_{\alpha}\right]$. Hence our assertion is proved. It is also clear that $T_{\infty}=F\left[G_{\infty}\right]$. But $G_{\infty}$ is an abelian group with $p$-primary component $G(p)_{d}$. Therefore $T_{\infty} \cong F\left[G(p)_{d} \times H\right]$ where $H$ has trivial $p$-primary component. In the same way, $T_{\infty}^{\prime} \cong F\left[G^{\prime}(p)_{d} \times H^{\prime}\right]$ and so $T_{\infty} \cong T_{\infty}^{\prime}$ implies $G(p)_{d} \cong$ $G^{\prime}(p)_{d}$.

Let $A$ be an indexing set and $\Gamma$ as before. Put $W=F\left[\sum_{A} \Gamma\right]$ where $F$ is some field of characteristic $p$. Let $B$ and $B^{\prime}$ be two subsets of $A$. Then the natural map 
$\sum_{A} \Gamma \rightarrow \sum_{A} \Gamma / \sum_{B} \Gamma$ induces a map $W \rightarrow F\left[\sum_{A} \Gamma / \sum_{B} \Gamma\right]$ with kernel, say $I$. Similarly, define $I^{\prime}$ by using $B^{\prime}$.

LEMMA 5. Let $W, I$, and $I^{\prime}$ be as above and assume there exists an F-automorphism $\varphi: W \rightarrow W$ such that $\varphi(I)=I^{\prime}$. Then $|B|=\left|B^{\prime}\right|$.

Proof. For each $a \in A$, let $X_{a}$ be an indeterminate. Let $L=F\left[\left\{X_{a}\right\}\right] /\left(\left\{X_{a}^{p}\right\}\right)$, i.e., $L$ is a truncated polynomial ring such that if $x_{a}$ is the natural image of $X_{a}$ in $L$, then $x_{a}^{p}=0$. Define $f: W \rightarrow L$ by requiring $f\left(g_{a}\right)=x_{a}+1$ for all $a \in A$. Then $f$ extends to $W$ and is well defined on $\sum_{A} \Gamma$ since $\left(x_{a}+1\right)^{p}=1$. Moreover, $f$ has an inverse defined by $f^{-1}\left(x_{a}\right)=g_{a}-e$, hence $f$ is an $F$-isomorphism.

Now $I$ is generated by $\left\{g_{a}-e \mid a \in B\right\}$. Therefore $f(I)$ is generated by $\left\{x_{a} \mid a \in B\right\}$. Similarly, $f\left(I^{\prime}\right)$ is generated by $\left\{x_{a} \mid a \in B^{\prime}\right\}$. Hence $\varphi$ induces an $F$-automorphism $\psi: L \rightarrow L$ such that $\psi(f(I))=f\left(I^{\prime}\right)$. But $L$ is a local ring with maximal ideal $M$ generated by $\left\{x_{a} \mid a \in A\right\}$. Since $\psi$ is an automorphism, we must have $\psi(M)=M$. Hence $f(I) / M f(I) \cong f\left(I^{\prime}\right) / M f\left(I^{\prime}\right)$ as $(L / M)$-modules. But $L / M \cong F$ by a natural isomorphism. We shall show that $\operatorname{dim}_{F}(f(I) / M f(I))=|B|$, hence, since a similar result applies to $I^{\prime}$, we shall have $|B|=\left|B^{\prime}\right|$.

Regarding $f(I)$ as an $F$-module, $f(I)$ has as basis, the monomials containing elements of $\left\{x_{a} \mid a \in B\right\}$ nontrivially. But $M f(I)$ has as basis, the monomials containing elements of $\left\{x_{a} \mid a \in B\right\}$ nontrivially and having degree at least two. Hence the monomials we lose in passing from $f(I)$ to $M f(I)$ are precisely $\left\{x_{a} \mid a \in B\right\}$. Therefore the dimension of the quotient space is $|B|$.

Let $F$ be a field of characteristic $p, T$ a commutative $F$-algebra, and $M$ a fixed ideal of $T$. For each ordinal number, $\alpha$, we want to define an $F$-algebra $S_{\alpha}$ and an ideal $I_{\alpha}$ of $S_{\alpha}$. We have previously defined $T_{\alpha}$ for all $\alpha$. Define $J_{\alpha}$ to be the ideal of $T_{\alpha}$ generated by $M \cap T_{\alpha+1}$. Put $S_{\alpha}=T_{\alpha} / J_{\alpha}$. Now let $K_{\alpha}$ be the kernel of $\theta: T_{\alpha} \rightarrow$ $T_{\alpha+1}$ and put $I_{\alpha}$ equal to the natural image of $K_{\alpha}$ in $S_{\alpha}$.

Proposition 5. Let $F$ be a perfect field of characteristic p. Let $G$ and $G^{\prime}$ be two abelian groups such that $F[G] \cong F\left[G^{\prime}\right]$ as $F$-algebras. Then the Ulm p-invariants of $G$ and $G^{\prime}$ are equal.

Proof. Put $T=F[G], T^{\prime}=F\left[G^{\prime}\right]$, and let $M$ and $M^{\prime}$ be the augmentation ideals of $T$ and $T^{\prime}$ respectively. Let $\varphi: T \rightarrow T^{\prime}$ be an isomorphism. We may assume that $\varphi(M)=M^{\prime}$. Now, as has been noted, by restriction, $\varphi$ induces an isomorphism $\varphi_{\alpha}: T_{\alpha} \rightarrow T_{\alpha}^{\prime}$ for all $\alpha$, which moreover satisfies $\varphi_{\alpha}\left(J_{\alpha}\right)=J_{\alpha}^{\prime}$ and $\varphi_{\alpha}\left(K_{\alpha}\right)=K_{\alpha}^{\prime}$. Hence we get a further induced isomorphism $\psi_{\alpha}: S_{\alpha} \rightarrow S_{\alpha}^{\prime}$ satisfying $\psi_{\alpha}\left(I_{\alpha}\right)=I_{\alpha}^{\prime}$.

We already know that $T_{\alpha}=F\left[G_{\alpha}\right]$ for all $\alpha$ and one observes that $J_{\alpha}$ is generated by the augmentation ideal of $F\left[G_{\alpha+1}\right]$. Therefore $S_{\alpha} \cong F\left[G_{\alpha} / G_{\alpha+1}\right]$ and the map $T_{\alpha} \rightarrow S_{\alpha}$ is induced by the natural map $G_{\alpha} \rightarrow G_{\alpha} / G_{\alpha+1}$. If we put $G_{\alpha}^{0}$ equal to the kernel of $\theta$ acting on $G_{\alpha}$, then $K_{\alpha}$ is generated by the augmentation ideal of $F\left[G_{\alpha}^{0}\right]$. Hence $J_{\alpha}+K_{\alpha}$ is generated by $\left\{g-e \mid g \in G_{\alpha+1} \cup G_{\alpha}^{0}\right\}$. It then follows that $J_{\alpha}+K_{\alpha}$ is generated by the augmentation ideal of $F\left[G_{\alpha+1} G_{\alpha}^{0}\right]$. Since $S_{\alpha} / I_{\alpha} \cong T_{\alpha} /\left(J_{\alpha}+K_{\alpha}\right)$, 
we see that $S_{\alpha} / I_{\alpha} \cong F\left[G_{\alpha} / G_{\alpha+1} G_{\alpha}^{0}\right]$ and that the map $S_{\alpha} \rightarrow S_{\alpha} / I_{\alpha}$ is induced by the passing from $G_{\alpha} / G_{\alpha+1}$ to the quotient group modulo the subgroup $\left(G_{\alpha+1} G_{\alpha}^{0}\right) / G_{\alpha+1}$. Similar statements apply to $T^{\prime}$.

Since $G_{\alpha} / G_{\alpha+1}$ may be regarded as a vector space over the integers modulo $p$, there is an isomorphism $G_{\alpha} / G_{\alpha+1} \cong \sum_{A} \Gamma$ for some indexing set $A$. But $S_{\alpha} \cong S_{\alpha}^{\prime}$ implies $\operatorname{dim}_{F} S_{\alpha}=\operatorname{dim}_{F} S_{\alpha}^{\prime}$ and hence $\left|G_{\alpha} / G_{\alpha+1}\right|=\left|G_{\alpha}^{\prime} / G_{\alpha+1}^{\prime}\right|$. Since the dimension of a vector space over a finite field is characterized by the cardinality of the vector space, we also have $G_{\alpha}^{\prime} / G_{\alpha+1}^{\prime} \cong \sum_{A} \Gamma$. If the isomorphisms are chosen properly, the natural maps $S_{\alpha} \rightarrow S_{\alpha} / I_{\alpha}$ and $S_{\alpha}^{\prime} \rightarrow S_{\alpha}^{\prime} / I_{\alpha}^{\prime}$ can be induced by dividing $\sum_{A} \Gamma$ by subspaces $\sum_{B} \Gamma$ and $\sum_{B^{\prime}} \Gamma$ respectively. Hence if $I$ and $I^{\prime}$ are as in Lemma 5 , there is an automorphism of $F\left[\sum_{A} \Gamma\right]$ taking $I$ into $I^{\prime}$. Hence $|B|=\left|B^{\prime}\right|$.

Finally, we observe that the Ulm $p$-invariant of $G$ corresponding to $\alpha$ is the dimension over the integers modulo $p$ of $G_{\alpha}^{0} /\left(G_{\alpha+1} \cap G_{\alpha}^{0}\right) \cong\left(G_{\alpha}^{0} G_{\alpha+1} / G_{\alpha+1}\right)$. But this dimension is just $|B|$. Similarly for $G^{\prime}$.

4. Final theorem. Let $R$ be a commutative ring with identity and $P$ a maximal ideal. Let $G$ and $H$ be two abelian groups such that $R[G] \cong R[H]$ as $R$-algebras. Let $F$ be an extension field of $R / P$. Then $F$ is naturally an $R$-algebra and moreover $F \otimes_{R} R[G] \cong F \otimes_{R} R[H]$. But this equation implies $F[G] \cong F[H]$ as $F$-algebras. We may of course take $F$ to be algebraically closed if desired. Moreover, if the prime $p$ is not invertible in $R$, then $p \in P$ for some maximal ideal, $P$. Hence, in this case we may further assume that $F$ has characteristic $p$.

THEOREM. Let $R$ be a commutative ring with identity and $G$ and $H$ abelian groups such that $R[G] \cong[H]$ as $R$-algebras. Let $G_{0}$ and $H_{0}$ be the respective torsion subgroups of $G$ and $H$. Then $G / G_{0} \cong H / H_{0}$. Moreover, $G$ is torsion-free if and only if $H$ is torsion-free. Now suppose the prime $p$ is not invertible in $R$. Let the p-primary components of $G$ and $H$, call them $G(p)$ and $H(p)$, be decomposed as $G(p) \cong G(p)_{d}$ $\times G(p)_{r}$ and $H(p) \cong H(p)_{d} \times H(p)_{r}$ where $G(p)_{d}$ and $H(p)_{d}$ are divisible, and $G(p)_{r}$ and $H(p)_{r}$ are reduced. Then $G(p)_{d} \cong H(p)_{d}$ and $G(p)_{r}$ and $H(p)_{r}$ have the same Ulm invariants.

Proof. We know $F[G] \cong F[H]$ as $F$-algebras for some algebraically closed field $F$, hence $G / G_{0} \cong H / H_{0}$ by Corollary 6 . Lemma 1 proves the statement on torsion-free. If $p$ is not invertible in $R$, we may assume $F$ has characteristic $p$, hence $G(p)_{d} \cong H(p)_{d}$ by Corollary 7. Finally, the Ulm $p$-invariants of $G$ and $H$ agree by Proposition 5, but they are the same as those of $G(p)_{r}$ and $H(p)_{r}$.

The rings over which the group algebra yields the most information are those in which none of the primes is invertible. For example, if we take $R$ to be the ring of integers in an algebraic number field, then the group algebras over $R$ of finitely generated abelian groups serve to classify the groups up to isomorphism. If $R$ is a field, however, we have little or no information on the torsion subgroup.

Here are two questions which immediately arise. First, we know that Ulm invariants of direct sums of countable reduced torsion groups serve to classify 
them. But, taking Corollary 3 into consideration, we might expect the algebra of an arbitrary uncountable group to contain more precise information than the Ulm invariants. Is it true that $G(p)_{r} \cong H(p)_{r}$ in the Theorem? The second question is whether the torsion subgroup of $G$ must be a direct summand if that of $H$ is?

\section{REFERENCES}

1. I. G. Connell, On the group ring, Canad. J. Math. 15 (1963), 650-685.

2. C. W. Curtis and J. Reiner, Representation theory of finite groups and associative algebras, Wiley, New York, 1962.

3. E. Deskins, Finite abelian groups with isomorphic group algebras, Duke Math. J. 23 (1956), 35-40.

4. G. Higman, The units of group rings, Proc. London Math. Soc. 46 (1940), 231-248.

5. K. W. Gruenberg, Resolutions by relations, J. London Math. Soc. 35 (1960), 481-494.

6. G. Losey, On dimension subgroups, Trans. Amer. Math. Soc. 97 (1960), 474-486.

7. M. Nagata, Local rings, Interscience, New York, 1962.

8. S. Perlis and G. Walker, Abelian group algebras of finite order, Trans. Amer. Math. Soc. 68 (1950), 420-426.

UNIVERSITY OF ROCHESTER,

ROCHESTER, NEW YORK 